\title{
Stand und Perspektiven des Qualitätsmanagements im schweizerischen Gesundheitswesen
}

Obschon das KVG in Art. 58 klare Regelungen und Aufträge enthält, sind Patientensicherheit und Qualitätsmanagement während etlicher Jahre nicht im Mittelpunkt der gesundheitspolitischen Diskussion unseres Landes gestanden. Verschiedene Vorfälle und Publikationen sowie entsprechende parlamentarische Vorstösse und die geplante Umsetzung des Projekts SwissDRG haben das Thema in jüngster Zeit wieder in den Vordergrund gerückt. Die Zeit für eine Standortbestimmung und einen Ausblick ist gekommen.

Dieser Aufgabe ist eine Tagung in Zürich gewidmet, an der zum einen die wichtigsten Partner des schweizerischen Gesundheitswesens in Referaten und an einer Podiumsdiskussion mitwirken. Um diese Gesamtsicht auszudrücken, steht sie unter dem gemeinsamen Patronat der FMH, von $\mathrm{H}+$, santésuisse und der SUVA. Dr. med. Peter Indra, MPH, Vizedirektor und Leiter des Direktionsbereichs Kranken- und Unfallversicherung des BAG, orientiert über die Absichten des Bundes zur Qualität in Schweizer Spitälern; Fritz Britt, Direktor santésuisse, referiert über die Erwartungen und eigenen Leistungen der Versicherer; Dr. phil. nat. Bernhard Wegmüller, Geschäftsführer $\mathrm{H}+$, spricht zum Thema «Qualitätssicherung: Verbände oder Wettbewerb - ein Widerspruch?». Weitere Referenten sind Prof. Dr. med. Dieter Conen, Direktor der Medizinischen Klinik des Kantonsspitals Aarau («Ist die Schweizer Medizin reif für die Qualitätstransparenz?»), Dr. med. Georg von Below, Leiter des Qualitätsmanagements am Spitalzentrum Biel/Bienne und Präsident der Schweizerischen Gesellschaft für Qualitätsmanagement im Gesundheitswesen SQMH («Stand der Qualitätssicherung in der Schweiz»), und Dr. rer. pol. Heinz Locher, Bern («Die Situation der Anbieter in der Schweiz»). Am zusammenfassenden Schlusspodium wirkt auch Dr. med. Pierre-François Cuénoud, Mitglied des
Zentralvorstandes der FMH, mit. Dieses steht unter der Leitung von Dr. Uwe Preusker, Berater und Publizist im Gesundheitswesen.

Damit der Stand der internationalen Diskussion und die anderswo gemachten Erfahrungen gezielt einbezogen werden können, wird die Tagung auch durch namhafte deutsche Referenten geprägt. So wird PD Dr. med. Thomas Mansky, Leiter der Medizinischen Entwicklung der HELIOS Kliniken, Berlin, über die Auswirkungen der DRG-Einführung auf die medizinische Qualität in verschiedenen Ländern informieren und dies am Beispiel der in Deutschland führenden HELIOS Kliniken illustrieren. Er und Dr. Wulf-Dietrich Leber vom AOK-Bundesverband, Bonn, werden zudem über das rasch auch in der Schweiz an Aktualität gewinnende Thema «Neue Methoden der Qualitätssicherung auf der Basis von Routinedaten» berichten. Dieser Ansatz ist gerade auch für die Ärzteschaft wegen der nicht zu Unrecht befürchteten zusätzlichen administrativen Belastung durch sonst stets zunehmende Sondererhebungen von hoher Bedeutung. Die beiden deutschen Referenten werden ebenfalls am Podiumsgespräch teilnehmen.

Eine Teilnahme wird sich für all diejenigen lohnen, die sich persönlich ein Bild der Lage verschaffen und eine eigene Standortbestimmung zum Thema vornehmen wollen.

\section{Stand und Perspektiven des Qualitäts- managements im schweizerischen Gesundheitswesen}

Veranstaltungsdatum: 6. Dezember 2007

Zeit: 9.00-18.00 Uhr

Veranstaltungsort: Kongresshaus Zürich

Veranstalter: RS Medical Consult $\mathrm{GmbH}$, Frauenberg 1, D-97980 Bad Mergentheim, E-Mail: info@rsmedicalconsult.com, Internet: www.rsmedicalconsult.com 